\title{
Upregulation of IncRNA RMRP promotes the activation of cardiac fibroblasts by regulating miR-613
}

\author{
SHUANG-YIN ZHANG ${ }^{*}$, SHENG-HUI HUANG ${ }^{*}$, SHI-XIONG GAO, \\ YING-BIN WANG, PING JIN and FENG-JIAO LU
}

Department of Anesthesiology, Lanzhou University Second Hospital, Lanzhou, Gansu 730030, P.R. China

Received April 3, 2019; Accepted July 25, 2019

DOI: $10.3892 / \mathrm{mmr} .2019 .10634$

\begin{abstract}
Long non-coding RNAs (lncRNAs) have been reported to serve a key role in a variety of cardiovascular diseases, including in cardiac fibrosis. The present study aimed to investigate the biological role and underlying mechanisms of the induction of cardiac fibroblasts by the IncRNA, RNA component of mitochondrial RNA processing endoribonuclease (RMRP). The results demonstrated that RMRP expression was upregulated in the presence of cardiac fibrosis in an abdominal aortic banding-treated rat model. Treatment with angiotensin II increased RMRP expression in cardiac fibroblasts, while the knockdown of RMRP by small-interfering RNA inhibited cardiac fibroblast proliferation, differentiation and collagen accumulation. To further investigate the underlying mechanisms of this interaction, microRNA (miR)-613 was predicted to be a target miR of RMRP and sequence alignment, luciferase activity and MS2 RNA immunoprecipitation were performed to detect the interaction between RMRP and miR-613. The results suggested that RMRP negatively regulated miR-613 in cardiac fibroblasts. Furthermore, miR-613 was indicated to mediate the promoting effect of RMRP on cardiac fibroblast activation. The current study suggested that RMRP promoted cardiac fibroblast activation by acting as a competing endogenous RNA for miR-613. Therefore, RMRP may be a novel target for the prevention or treatment of cardiac fibrosis.
\end{abstract}

\section{Introduction}

Cardiac fibrosis is the process of increased cardiac fibroblast activity that leads to the over-production of excessive

Correspondence to: Dr Shuang-Yin Zhang, Department of Anesthesiology, Lanzhou University Second Hospital, 82 Cuiyingmen, Lanzhou, Gansu 730030, P.R. China

E-mail: shyzhlanzhou@sina.com

*Contributed equally

Key words: cardiac fibrosis, cardiac fibroblasts, long noncoding RNA, microRNA extracellular matrix (ECM) within the myocardium (1). Under normal conditions, the ECM undergoes a balanced turnover via the degradation and synthesis of its constituent proteins, which include collagens. However, this balance is disturbed under pathological conditions of the heart, such as increased loading (e.g., in hypertension) or injury (e.g., during myocardial infarction). This imbalance results in the excessive deposition of ECM, which can profoundly affect cardiac function by impairing electrical conduction and increasing myocardial stiffness, which are both common risk factors for heart failure and arrhythmias (2). Cardiac fibroblasts are responsible for regulating the synthesis and degradation of the ECM. Under pathological conditions, activated cardiac fibroblasts can exhibit increased proliferation and differentiation into myofibroblasts that express contractile proteins, including $\alpha$-smooth muscle actin ( $\alpha$-SMA), and increase ECM accumulation (3). Therefore, determining the underlying mechanisms by which the activation of cardiac fibroblasts is regulated, may provide novel insights in the prevention and treatment of heart failure.

It has been previously reported that $1.5-2.0 \%$ of the human genome codes for proteins, but primary RNA transcripts are present in $\sim 70-80 \%$ (4). A growing body of evidence has identified non-coding RNAs (ncRNAs) as possible modulators of cardiac fibrosis. microRNAs (miRNAs/miRs) are defined as small ncRNA molecules (containing 22 nucleotides) and have been reported to regulate cardiac fibroblast activation in cardiac fibrosis (5). In addition to miRNAs, long noncoding RNAs (lncRNAs) serve roles in numerous cellular processes, including those present in cardiovascular disease. A number of lncRNAs have been identified in the heart, but only a small number have been functionally characterized (6). RNA component of mitochondrial RNA processing endoribonuclease (RMRP) is a long non-coding RNA that is expressed in a variety of murine and human tissues (7). RMRP encodes the RNA component of mitochondrial RNA processing endoribonuclease, which cleaves mitochondrial RNA at the priming site of DNA replication. RMRP has also been revealed to interact with the telomerase reverse transcriptase catalytic subunit to form a distinct ribonucleoprotein complex, which exhibits RNA-dependent RNA polymerase activity and produces double-stranded RNAs that can be processed into small interfering RNA (siRNA) (7). Previous studies have investigated the role of RMRP in numerous cancer types, including lung cancer (8), gastric cancer (9) and glioma (10). Furthermore, 
Wang et al (11) demonstrated that higher RMRP expression in nucleus pulposus tissues was associated with disc degeneration grade. Another study has indicated that RMRP overexpression increased nucleus pulposus cell growth and regulated ECM expression by targeting miR-206 (11). Recently, Greco et al (12) profiled the expression of 83 lncRNAs, which are known to be associated with numerous human diseases, in the left ventricle biopsies of patients with heart failure and demonstrated that RMRP was significantly upregulated in these patients. Steinbusch et al (13) indicated that RMRP was associated with chondrocyte hypertrophy and determined chondrogenic differentiation, suggesting that RMRP served a role in the regulation of the dynamic balance of ECM degradation and synthesis. In consideration of the key role of cardiac fibrosis in heart failure, the current study speculated that RMRP may be involved in regulating the activation of cardiac fibroblasts.

The current study investigated the biological role of the lncRNA RMRP in the activation of cardiac fibroblasts during cardiac fibrosis. The results demonstrated the aberrant expression of RMRP in the fibrotic myocardium and investigated its role in cardiac fibroblast activation, which was characterized as an increase of proliferation, differentiation and collagen accumulation. It has been previously demonstrated that lncRNAs act as ceRNAs when binding with microRNAs and that numerous microRNAs serve crucial functions in cardiac fibrosis (5). In the current study, whether RMRP interacted with microRNA during cardiac fibroblast activation was further determined.

\section{Materials and methods}

Animals. Specific pathogen-free Sprague-Dawley (SD) rats $(\mathrm{n}=16$, male, 6-8 weeks old) weighing between 150-180 g were provided by Beijing HFK Bioscience Co., Ltd. The animals were housed at $24 \pm 2^{\circ} \mathrm{C}, 60 \pm 10 \%$ relative humidity with a 12-h light/dark cycle. The rats were acclimated to the laboratory conditions; standard rat chow and water were available ad libitum. All animal experiments performed in the current study were approved by The Animal Care and Use Committee of Lanzhou University (Lanzhou, China). The rats were randomly divided into two groups, each of which included 8 rats: The sham-operated group (Sham) and the abdominal aortic banding (AAB) group. Myocardial fibrosis was induced by AAB for 6 weeks (14). After 6 weeks, all rats were anesthetized using an intraperitoneal injection of chloral hydrate $(10 \%$; $350 \mathrm{mg} / \mathrm{kg}$ ). No signs of peritonitis, pain or discomfort were observed. The survival of rats was confirmed by monitoring their breathing and heartbeat. Subsequently, the rats were sacrificed using cervical dislocation. After sacrifice, the left ventricle was dissected from the heart. Part of the isolated left ventricles were used for Masson's trichrome staining (10 $\mathrm{min}$ at room temperature) after fixation in $4 \%$ paraformaldehyde for $24 \mathrm{~h}$ at room temperature. The images (magnification, x200) were observed by using a light microscope (Olympus Corporation). The remaining samples were used to determine the expression of $\alpha$-SMA, collagen and lncRNA RMRP expression.

Cardiac fibroblast isolation. Primary cardiac fibroblasts were isolated from the myocardium of newborn SD rats (1-2 days old) as previously described (15). Briefly, shredded myocardium was digested with $0.1 \%$ pancreatin at $37^{\circ} \mathrm{C}$. Cardiac fibroblasts were separated from cardiomyocytes using differential adhesion. Isolated cardiac fibroblasts were cultured at $37^{\circ} \mathrm{C}$ under $5 \% \mathrm{CO}_{2}$ in DMEM (Gibco; Thermo Fisher Scientific, Inc.) supplemented with 10\% FBS (Gibco; Thermo Fisher Scientific, Inc.), $100 \mathrm{U} / \mathrm{ml}$ penicillin and $100 \mathrm{~g} / \mathrm{ml}$ streptomycin. The activated cardiac fibroblasts were induced using $10^{-5} \mathrm{mM}$ angiotensin-II for $24 \mathrm{~h}$.

Cell viability assay. Cardiac fibroblasts $\left(5 \times 10^{3} / \mathrm{ml}\right)$ were cultured in 96-well plates. After treatment, cell viability was determined using a cellTiter $966^{\circledR}$ AQueous one solution cell proliferation assay kit (Promega Corporation). The assay was performed according to manufacturer's protocol.

EdU incorporation assay. The EdU incorporation assay kit was used to measure the proliferative ability of cardiac fibroblasts and was purchased from Guangzhou Ribobio Co., Ltd. The proliferative cells that had incorporated EdU showed green fluorescence. Cell nuclei that were stained using DAPI for $10 \mathrm{~min}$ at $25^{\circ} \mathrm{C}$ showed blue fluorescence. Data are presented as the ratio of EdU-positive cells (green cells) to the total DAPI-positive cells (blue cells).

Reverse transcription-quantitative $(R T-q) P C R$. Total RNA samples were extracted using TRIzol ${ }^{\circledR}$ reagent (Invitrogen; Thermo Fisher Scientific, Inc.). The RNA was converted to cDNA using an RT kit (Applied Biosystems; Thermo Fisher Scientific, Inc.), according to the manufacturer's protocol. The protocol for RT was as follows: $37^{\circ} \mathrm{C}$ for $60 \mathrm{~min}, 85^{\circ} \mathrm{C}$ for $5 \mathrm{~min}$ and then hold at $4^{\circ} \mathrm{C}$. The cDNA was amplified using the $\mathrm{SYBR}^{\circledR}$ Green method to determine RMRP, $\alpha$-SMA, Collagen-I, miR-613 and miR-1 expression, which were normalized to GAPDH or U6. RT-qPCR analysis was performed using a SYBR Green PCR Master Mix kit (Takara Bio, Inc.) with an ABI 7500 Thermocycler (Applied Biosystems, Foster City, CA, USA). PCR cycles were as follows: 10 min pretreatment at $95^{\circ} \mathrm{C}$, $93^{\circ} \mathrm{C}$ for $15 \mathrm{sec}, 67^{\circ} \mathrm{C}$ for $45 \mathrm{sec}(45$ cycles $), 93^{\circ} \mathrm{C}$ for $15 \mathrm{sec}, 67^{\circ} \mathrm{C}$ for $1 \mathrm{~min}, 95^{\circ} \mathrm{C}$ for $15 \mathrm{sec}$, a final extension at $75^{\circ} \mathrm{C}$ for $10 \mathrm{~min}$ and held at $4^{\circ} \mathrm{C}$. mRNA expression was quantified via the $2^{-\Delta \Delta \mathrm{Cq}}$ method (16). The primer sequences are listed in Table SI.

Cell transfection. The siRNA sequences (RMRP siRNA and miR-613 inhibitor, $50 \mathrm{nM}$ ) and the miR-613 mimic were supplied by Guangzhou RiboBio Co., Ltd. The pcDNA3.1-RMRP and empty pcDNA3.1 were supplied and synthesized by Shanghai GenePharma Co., Ltd. Transfection was performed using Lipofectamine ${ }^{\circledR} 2000$ (Invitrogen; Thermo Fisher Scientific, Inc.), according to the manufacturer's protocol. The cells were harvested $24 \mathrm{~h}$ after transfection and then used for subsequent experiments. The sequences were as follows: RMRP siRNA, 5'-CCUAGGCUACACACUGAGGACUTT-3'; RMRP siRNA NC, 5'-UUCUCCGAACGUGUCACGUTT-3'; miR-613 mimic, 5'-CAAGUGUGAAGGGACCCUUCC-3'; miR-613 inhibitor, 5'-CCUUCCUGUAGUGUCUUAU-3' and miR-613 inhibitor NC, 5'-GATGGTCTTGCGGTCGTAGAT-3'

Western blot analysis. Total protein was extracted from the myocardium and fibroblasts using RIPA buffer (containing 
$0.1 \%$ PMSF). Protein concentration was measured using Bradford Protein Assay kit (Bio-Rad Laboratories, Inc.). Equal amounts $(20 \mu \mathrm{g} /$ lane $)$ of protein were fractionated using 10\% SDS-PAGE and transferred to PVDF membranes. The membranes were blocked with $5 \%$ skimmed milk at room temperature for $1 \mathrm{~h}$. Blotting was then probed with $\alpha$-SMA (1:2,000; cat. no. ab7817; Abcam) and GAPDH (1:2,000; cat. no. sc-137179; Santa Cruz Biotechnology, Inc.) primary antibodies. GAPDH was used as an internal reference. The chemiluminescence signals were captured using an EasySee Western Blot kit (Beijing TransGen Biotech Co., Ltd.) and analyzed using Image J 1.43 (National Institutes of Health).

Measurement of collagen content. Measurements of the collagen content in cardiac fibroblasts were performed according to a previous report (17). Tris buffer ( $\mathrm{pH} 7.5$ ) was used to lyse cardiac fibroblasts and picrosirius red (dissolved in saturated picric acid) was added to extract the collagen. Collagen content was calculated using the linear calibration curve generated from standards and normalized to the total protein content.

Luciferase reporterassay. The miRcode algorithm(http://www. mircode.org/) was used to predict the RMRP-miRNA interaction, the result showed miR-613 may interact with RMRP. Therefore, luciferase reporter assay was performed to determine the interaction between RMRP and miR-613. The fragment of RMRP containing the target sequence of miR-613 was inserted into a pmirGLO dual-luciferase miRNA target expression vector (Guangzhou RiboBio Co., Ltd.) to form the reporter vector RMRP wild-type (RMRP-WT), while the RMRP mutated type (RMRP-MUT) contained a mutated binding site. The RMRP-WT or the RMRP-MUT reporter plasmid were cotransfected, with miR-613 mimic or miR-NC, into cardiac fibroblasts using Lipofectamine 2000. Luciferase activity was measured using a dual luciferase reporter assay system (Guangzhou RiboBio Co., Ltd.). Luciferase activity was normalized to Renilla luciferase activity.

RNA immunoprecipitation. Cardiac fibroblasts were cotransfected with pcDNA-MS2, pcDNA-RMRP-WT-MS2, or pcDNA-RMRP-MUT-MS2 (Shanghai GeneChem Co., Ltd.). After a period of $48 \mathrm{~h}$, RNA RIPA assays were performed on cells using an anti-green fluorescent protein (GFP) antibody (1:1,000, ab13970, Abcam) and the EZ-Magna RIP ${ }^{\mathrm{TM}}$ RNA-Binding Protein Immunoprecipitation kit (EMD Millipore), according to the manufacturer's protocol.

Statistical analysis. The data are presented as the mean \pm standard error of the mean. All experiments were performed three times. Statistical significance was determined using either an unpaired Student's t-test for two comparisons or an analysis of variance followed by the Student-Newman-Keuls test for multiple comparisons. Data were analyzed using SPSS version 23.0 (IBM Corp.). $\mathrm{P}<0.05$ was considered to indicate a statistically significant difference.

\section{Results}

Expression of IncRNA RMRP is upregulated in cardiac fibrosis. Rats subjected to AAB surgery for 6 weeks exhibited cardiac fibrosis, as demonstrated by an increased collagen accumulation in the myocardial tissue (Fig. 1A) and upregulated $\alpha$-SMA and collagen mRNA and protein expression (Fig. 1B and C). In the fibrotic myocardium, RMRP expression was significantly upregulated $(\mathrm{P}<0.01$; Fig. 1D). Cardiac fibroblasts and cardiomyocytes are widely considered to be the two most common cardiac cell types, accounting for $90 \%$ of cardiac cells (18). Therefore, primary cardiac fibroblasts and cardiomyocytes were isolated from rat hearts to determine RMRP expression. The results demonstrated that RMRP expression exhibited a significantly increased upregulation in the cardiac fibroblasts and cardiomyocytes of the AAB group compared with the sham group $(\mathrm{P}<0.01$; Fig. $1 \mathrm{E})$. Similar results were obtained from the in vitro study, which indicated that RMRP was significantly upregulated in the Ang II-treated cardiac fibroblasts and cardiomyocytes $(\mathrm{P}<0.05$; Fig. 1F). Furthermore, the upregulation of RMRP in cardiac fibroblasts exhibited greater significance than the upregulation in cardiomyocytes, which led to the speculation that RMRP may serve a key role in cardiac fibroblast activation during cardiac fibrosis.

RMRP knockdown inhibits the cardiac fibroblast activation induced by Ang-II. The activation of cardiac fibroblasts is associated with the development of cardiac fibrosis. Ang-II and TGF- $\beta 1$ myocardial levels are increased in a number of pathologies that are characterized by myocardial remodeling and fibrosis. Ang-II and TGF- $\beta 1$ have been indicated to promote cardiac fibroblast activation, which serve a key role in cardiac fibrosis (19). Therefore, Ang-II and TGF- $\beta 1$ were used to induce the activation of cardiac fibroblasts in the present study and the results demonstrated that RMRP expression was upregulated in cardiac fibroblasts treated with Ang-II and TGF- $\beta 1$. The significant different between Ang-II and the control was greater than that of TGF- $\beta 1$ (Fig. 2A). Therefore, Ang-II was used to induce cardiac fibroblast activation in the current study.

To investigate the role of RMRP in cardiac fibrosis, the effect of RMRP knockdown on Ang-II-induced cardiac fibroblast activation was investigated. As presented in Fig. 2B, RMRP siRNA was indicated to significantly downregulate RMRP expression $(\mathrm{P}<0.01)$, suggesting the successful knockdown of RMRP. Furthermore, RMRP siRNA inhibited the Ang-II-induced cardiac fibroblast proliferation (Fig. 2C and D). In addition to proliferation, differentiation and collagen accumulation were also indicated to be important pathological features in the activation of cardiac fibroblasts. According to a previous study (19), $\alpha$-SMA and collagen mRNA and protein expression were upregulated in cardiac fibroblasts following Ang-II treatment. However, the effects of Ang-II were also reversed by RMRP siRNA, which suggested that RMRP knockdown inhibited the Ang-II-induced differentiation and collagen accumulation of cardiac fibroblasts (Fig. 2E-G). Collectively, these results indicated that RMRP knockdown inhibited Ang-II-induced cardiac fibroblast activation.

RMRP interacts with miR-613 in cardiac fibroblasts. To investigate the interaction between RMRP and miRNAs, the miRcode algorithm was used to predict the RMRP-miRNA interaction. The results demonstrated that RMRP contained 
A

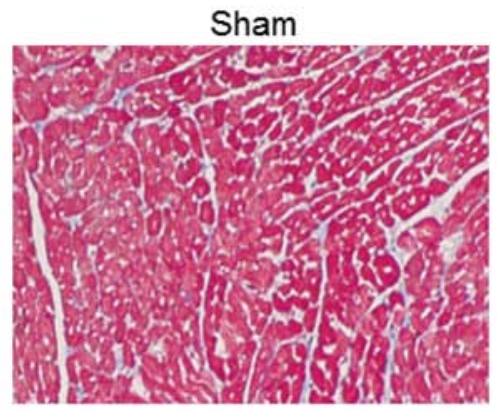

C

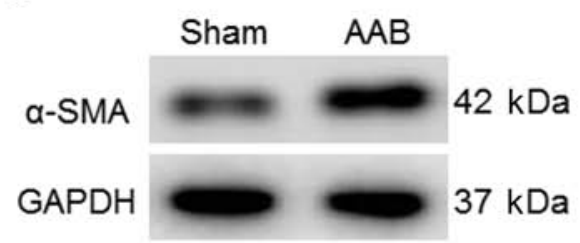

$A A B$

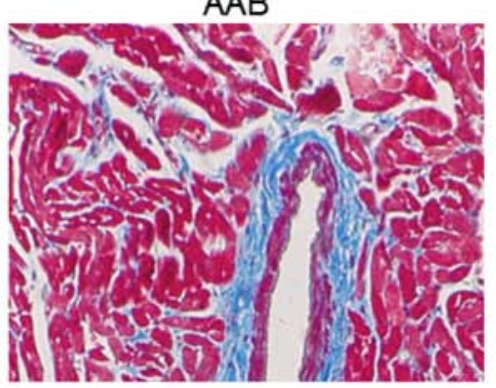

B

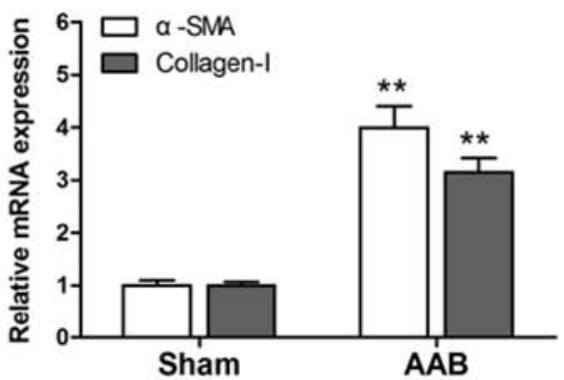

D

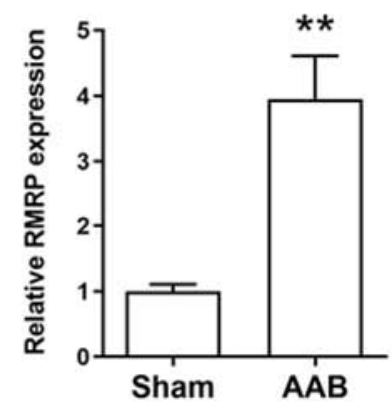

E

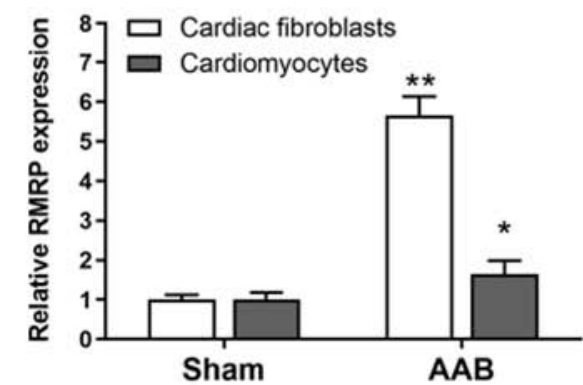

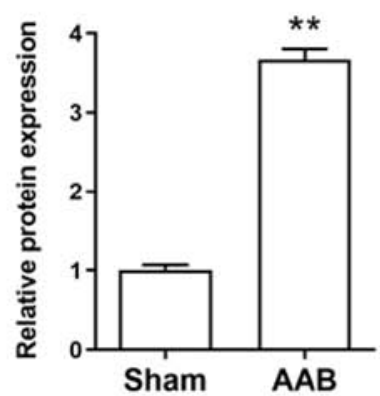

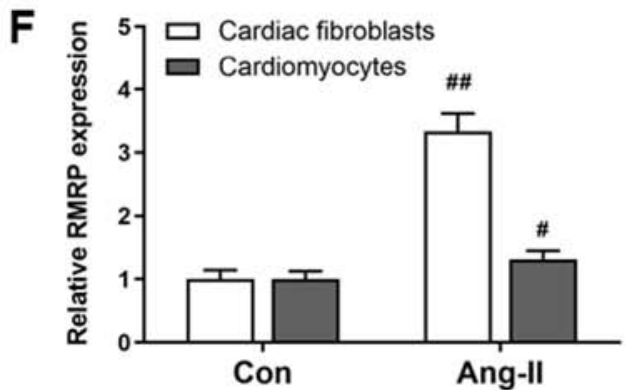

Figure 1. The expression of lncRNA RMRP in AAB-induced cardiac fibrosis. (A) Accumulation of collagen was assessed by Masson staining (magnification, $\mathrm{x} 200$ ). (B) The mRNA expression of $\alpha$-SMA and Collagen I in myocardial tissue was measured using RT-qPCR. (C) The protein expression of $\alpha$-SMA in myocardial tissue was determined using western blotting. (D) The expression of RMRP in myocardial tissue was assessed using RT-qPCR. (E) The expression of RMRP in cardiac fibroblasts and cardiomyocytes isolated from rat hearts was measured using RT-qPCR. Data are presented as the mean \pm SEM. $n=8$ animals for each group. ${ }^{*} \mathrm{P}<0.05,{ }^{* *} \mathrm{P}<0.01$ vs. Sham. (F) The expression of RMRP in Ang-II-treated cardiac fibroblasts and cardiomyocytes was measured using RT-qPCR. Data are mean \pm SEM. $n=3$ independent experiments. ${ }^{\#} \mathrm{P}<0.05$, ${ }^{\# \#} \mathrm{P}<0.01$ vs. Con. AAB, abdominal aortic banding; SEM, standard error of the mean; RT-q, reverse transcription-quantitative; Con, control; $\alpha$-SMA, $\alpha$-smooth muscle actin; RMRP, RNA component of mitochondrial RNA processing endoribonuclease; Ang II, angiotensin II.

one seed sequence that may bind six miRNAs, including miR-1, miR-206, miR-613, miR-122, miR-122a and miR-1352 (Fig. 3A). Among the predicted target miRNAs, miR-613 and miR-1 have been reported to be closely associated with myocardial I/R injury and cardiac hypertrophy, respectively $(20,21)$. Furthermore, miR-613 and miR-1 expression was downregulated in Ang-II treated cardiac fibroblasts (Fig. 3B). RMRP knockdown by siRNA upregulated miR-613 expression but did not affect miR-1 expression. Therefore, it was suggested that RMRP interacted with miR-613 in cardiac fibroblasts. As presented in Fig. 3B, Ang-II downregulated miR-613 expression, which was reversed by RMRP siRNA, suggesting that RMRP regulated miR-613. To further explore the interaction between RMRP and miR-613, a luciferase reporter and MS2 RIP assays were performed. The miR-613 binding site of RMRP was predicted, as presented in Fig. 3C and RMRP-MUT was designed according to this binding site. A luciferase reporter assay containing RMRP-WT or RMRP-MUT was constructed and the results revealed that miR-613 overexpression decreased RMRP-WT activity more so than RMRP-MUT (Fig. 3D). To validate the direct binding between miR-613 and RMRP, MS2 RIP was used to isolate the endogenous miRNAs that are associated with RMRP. The results revealed that miR-613 was significantly upregulated (Fig. 3E and F). These results suggested that RMRP interacts with miR-613 in cardiac fibroblasts.

miR-613 mediates the promoting effect of RMRP on cardiac fibroblast activation. To further investigate whether miR-613 mediated the effect of RMRP on cardiac fibroblast activation, an miR-613 inhibitor was used to inhibit miR-613 expression in cardiac fibroblasts treated with Ang-II and RMRP siRNA. The results demonstrated that RMRP siRNA inhibited Ang-II-induced cardiac fibroblast proliferation, differentiation 


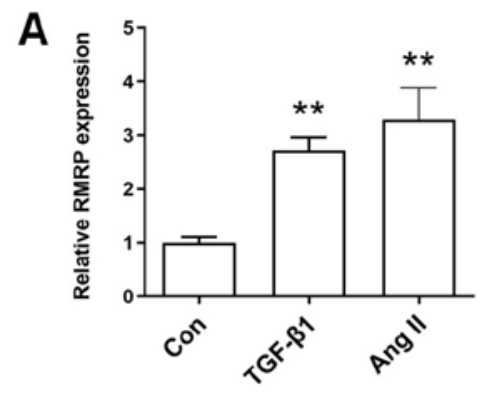

B

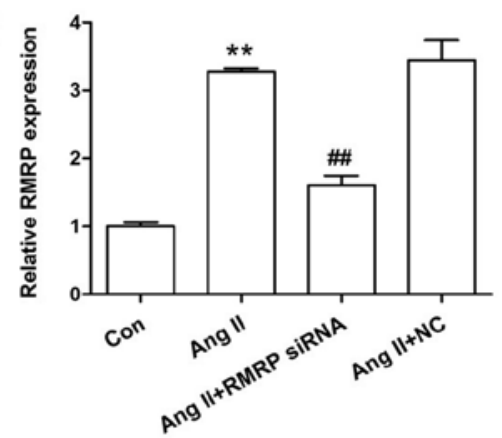

D
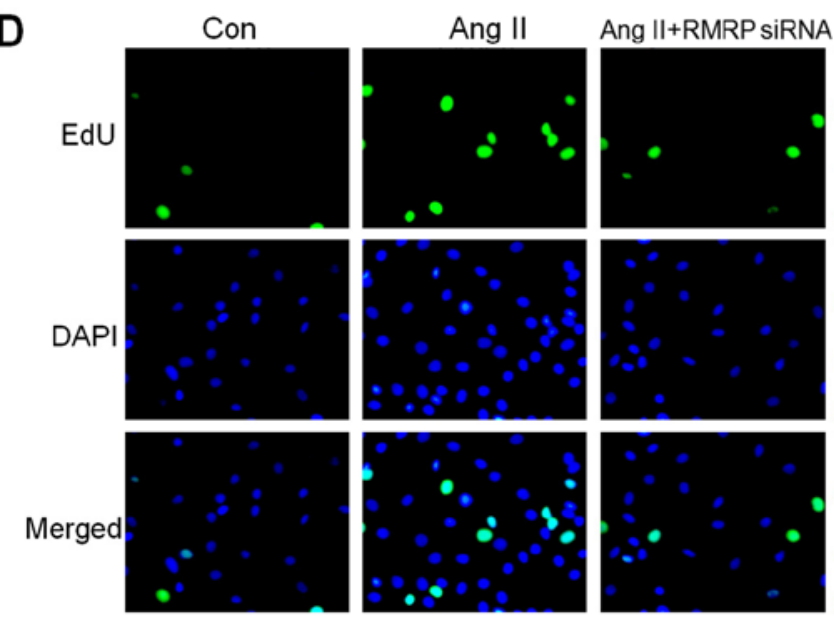

E

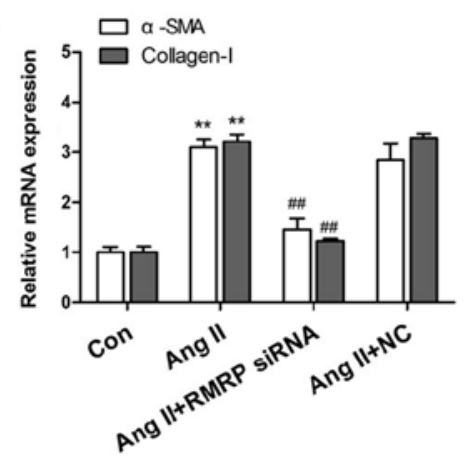

F

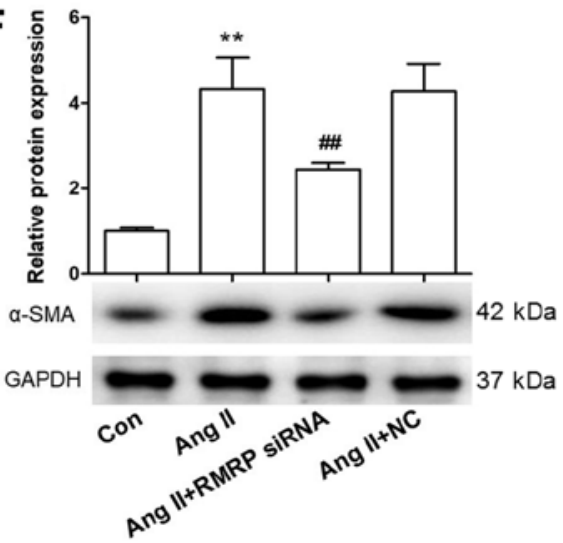

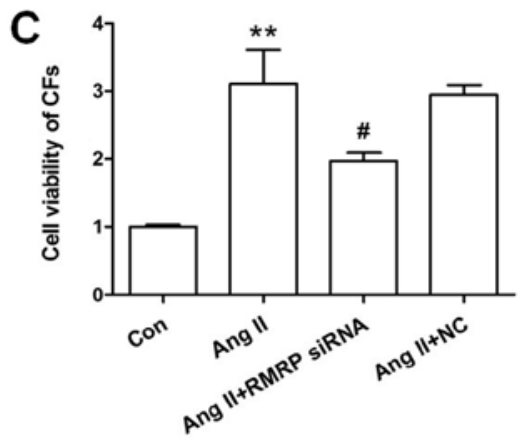

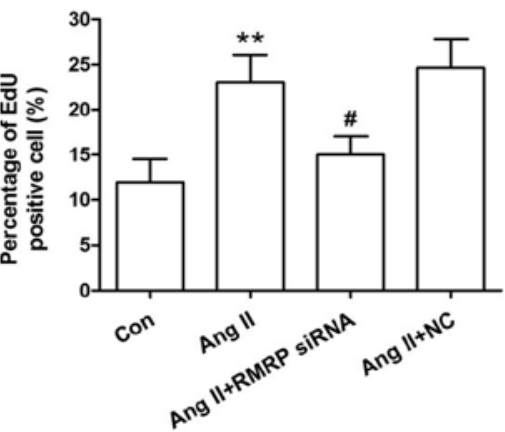

G

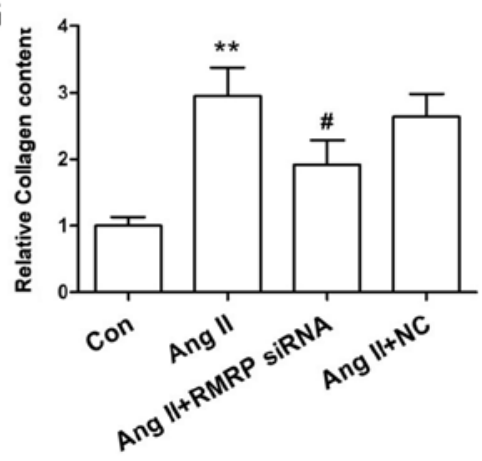

Figure 2. The effect of RMRP knockdown on the Ang-II-induced cardiac fibroblasts activation. (A) The effect of Ang-II and TGF- $\beta 1$ on the RMRP expression of cardiac fibroblasts. The effect of RMRP siRNA on the (B) RMRP expression, (C) cell viability, (D) proliferation, (E) mRNA and (F) protein expression of $\alpha$-SMA and $(G)$ Collagen of Ang-II-treated cardiac fibroblasts. Magnification, $x 400$ for the EdU images of D. Data are mean \pm standard error of the mean. $\mathrm{n}=3$ independent experiments. ${ }^{* *} \mathrm{P}<0.01$ vs. Con; ${ }^{*} \mathrm{P}<0.05$ and ${ }^{\# \#} \mathrm{P}<0.01$ vs. Ang-II. NC, RMRP siRNA negative control; si, small interfering; Con, control; TGD, transforming growth factor; $\alpha$-SMA, $\alpha$-smooth muscle actin; RMRP, RNA component of mitochondrial RNA processing endoribonuclease; Ang II, angiotensin II.

and collagen accumulation, while miR-613 inhibitor reversed the effect of RMRP siRNA (Fig. 4A-F). These results suggested that RMRP promoted cardiac fibroblast activation by downregulating miR-613.

\section{Discussion}

New sequencing technologies, combined with bioinformatics and computational tools, have transformed the traditional view of ncRNA as the 'junk of the genome', with the appreciation that these transcripts may influence disease-associated genes and contribute to disease pathogenesis (22). ncRNAs are classified into two groups: Small ncRNAs ( $\leq 200$ nucleotides long) and lncRNAs (>200 nucleotides). Small ncRNAs, which include miRNAs, have been investigated for a number of years, while lncRNA studies have only started recently. In recent years, it has become increasingly evident that lncRNAs serve a key role in the posttranscriptional, epigenetic and translational coordination of gene expression in developmental and disease processes (23). IncRNAs have been reported to have implications for cancer biology (24). Furthermore, increasing evidence has demonstrated the roles of lncRNAs 
A

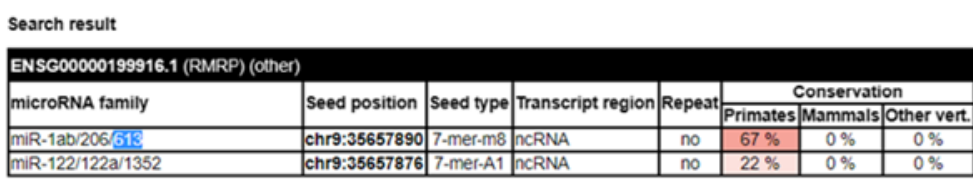

C
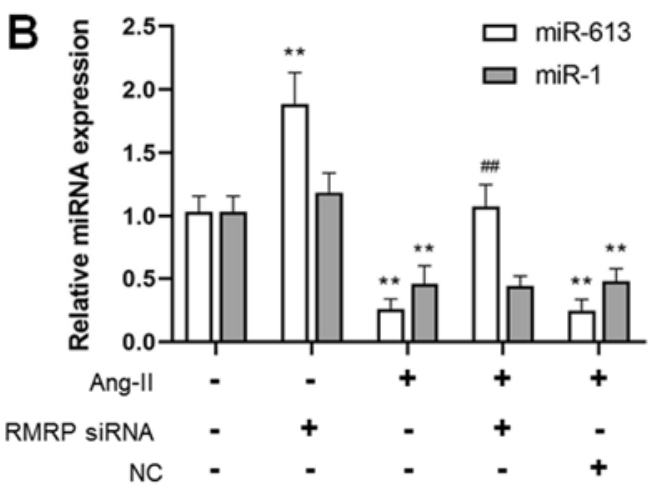

D

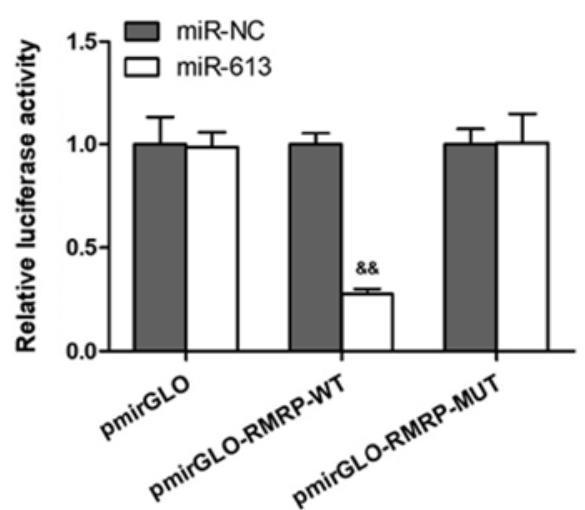

$\mathbf{F}$

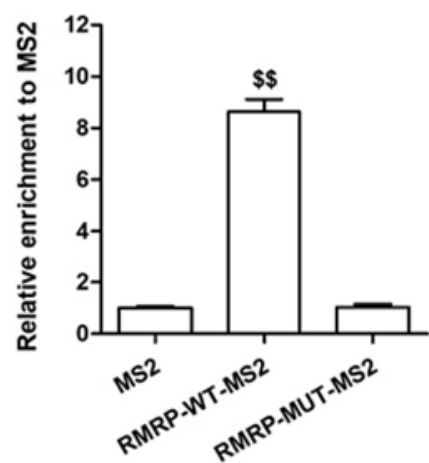

Figure 3. RMRP interacts with miR-613 in cardiac fibroblasts. (A) An miRcode algorithm was used to predict RMRP-miRNA interaction. RMRP contains one seed sequence that can combine 6 miRNAs. (B) Effect of RMRP siRNA on the miR-613 and miR-1 expression in Ang-II-treated cardiac fibroblasts was assessed using reverse transcription-quantitative PCR. (C) Sequence alignment showing the complementarity between RMRP and miR-613. 'I' indicates base pairing. (D) Luciferase activity in cardiac fibroblasts co-transfected miR-613 mimic and luciferase reporters containing RMRP. (E) A diagram and the $(\mathrm{F})$ results of MS2 RIP, performed to detect miR-613 endogenously associated with RMRP. Data are presented as the mean \pm standard error of the mean. $\mathrm{n}=3$ independent experiments. ${ }^{* *} \mathrm{P}<0.01$ vs. Con; ${ }^{\#} \mathrm{P}<0.01 \mathrm{vs}$. Ang-II; ${ }^{\&} \mathrm{P}<0.01$ vs. pmirGLO; ${ }^{\$ \$} \mathrm{P}<0.01$ vs. MS2. miRNA, microRNA; siRNA, small interfering RNA; GFP, green fluorescent protein; Con, control; RMRP, mitochondrial RNA processing endoribonuclease; WT, wild-type; MUT, mutant; RIP, radioimmunoprecipitation; Ang II, angiotensin II.

in a variety of biological processes. Cardiovascular disease is a research area of increasing interest. The expression of lncRNAs has been revealed to be associated with numerous cardiovascular conditions and IncRNAs have been indicated to be involved in regulating the development of cardiovascular diseases, including pathological hypertrophy, vascular disease, atherosclerosis, dyslipidemia and metabolic disorder (6).

Recent studies have demonstrated the mechanistic function of lncRNAs in cardiac fibroblast activation and cardiac fibrosis (5). Tao et al (25) demonstrated that lncRNA GAS5 was downregulated in cardiac fibrosis tissues and activated cardiac fibroblasts, while the overexpression of GAS5 inhibited cardiac fibroblast proliferation. In a mouse model of myocardial infarction, IncRNA MIAT was upregulated during cardiac interstitial fibrosis. MIAT knockdown has been revealed to reduce cardiac fibrosis (26). Consistent with this in vivo study, MIAT expression was upregulated in activated cardiac fibroblasts that were induced by Ang-II, whereas MIAT knockdown abrogated the fibrogenesis (26). Similarly, the upregulated lncRNA n379519 and PFL exhibited pro-fibrotic effects during myocardial infarction $(27,28)$. In the present study, RMRP expression was upregulated and accompanied by cardiac fibrosis in an AAB-treated rat model. This was also determined in cardiac fibroblasts that were isolated from the fibrotic myocardium 
A
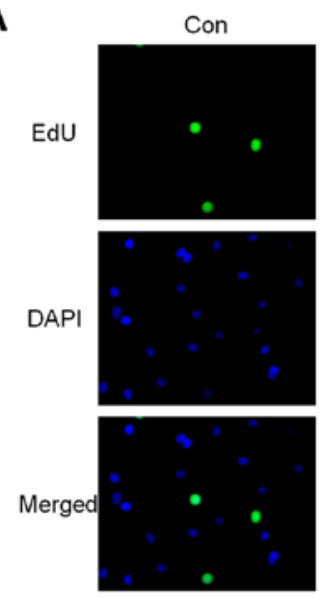

B

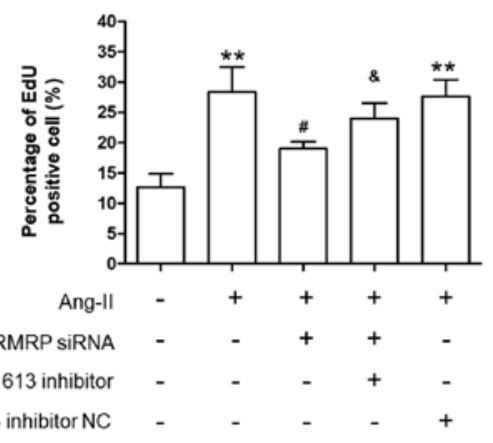

D

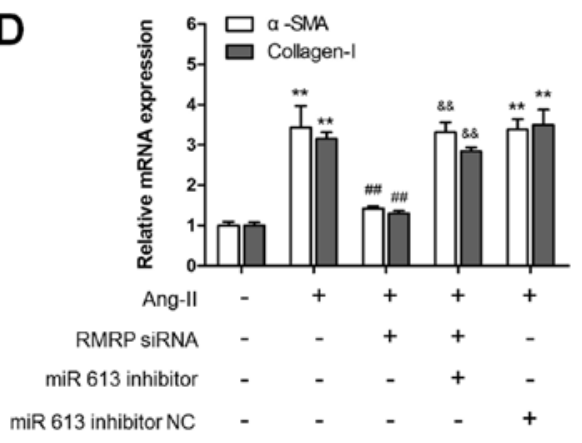

$\mathbf{F}$
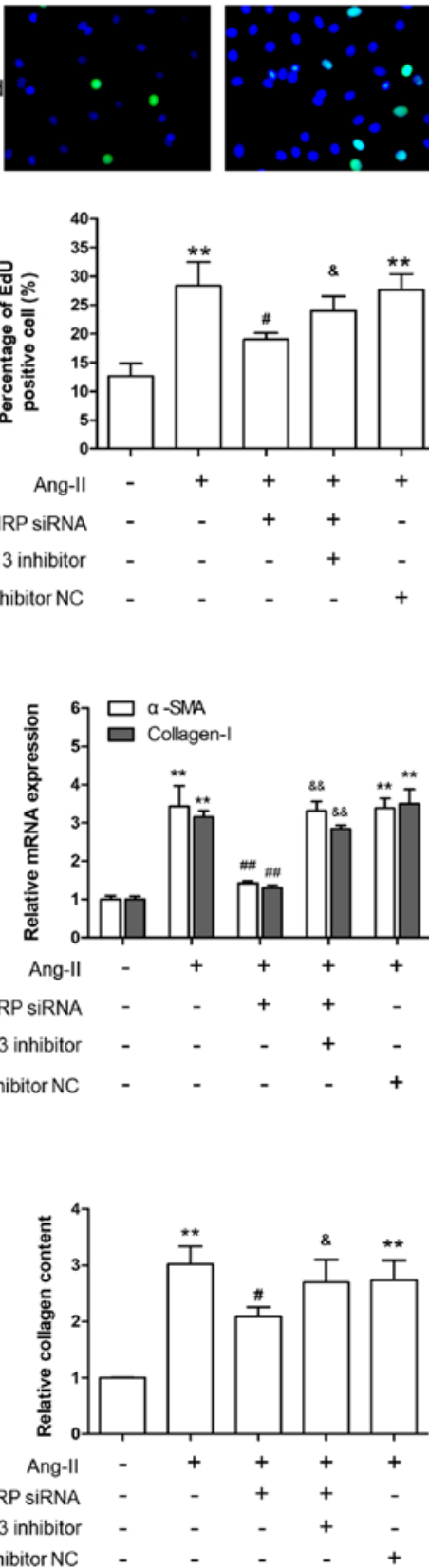

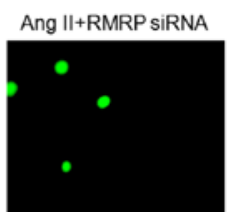

Ang II+RMRP siRNA

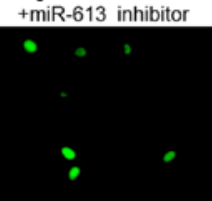

Ang $\|+m i R-613$

Inhibitor NC
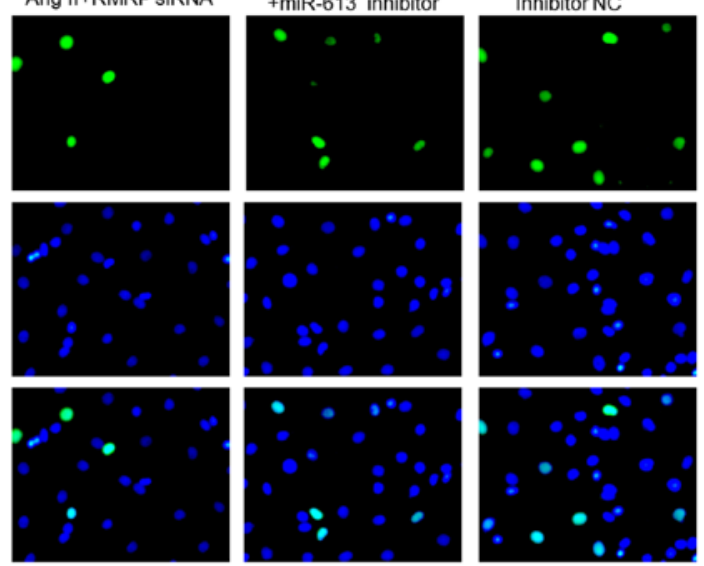

C
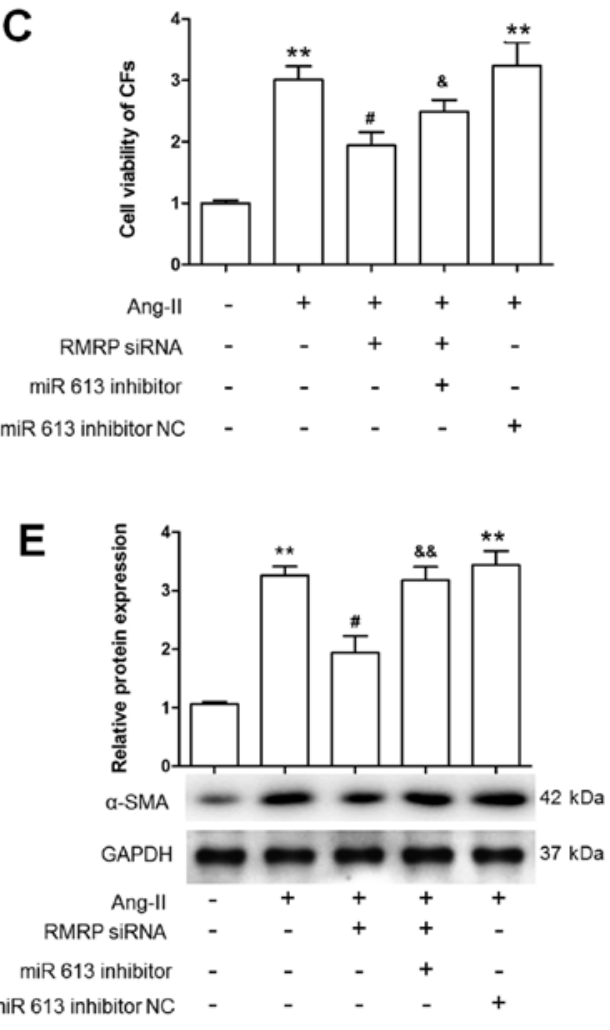

Figure 4. RMRP promotes cardiac fibroblasts activation through miR-613. The effect of miR-613 inhibitor on the proliferation as shown by (A) EdU staining and (B) graphical analysis (C) cell viability, $\alpha$-SMA and Collagen (D) mRNA and (E) protein expression (F) of cardiac fibroblasts treated with Ang-II and RMRP siRNA. Magnification, $x 400$ for the EdU images of A. miR-613 inhibitor NC, miR-613 inhibitor negative control. Data are mean \pm standard error of the mean. $\mathrm{n}=3$ independent experiments. ${ }^{* *} \mathrm{P}<0.01$ vs. Con; ${ }^{\#} \mathrm{P}<0.05$ and ${ }^{\# \#} \mathrm{P}<0.01$ vs. Ang-II; ${ }^{*} \mathrm{P}<0.05$ and ${ }^{\& \&} \mathrm{P}<0.01$ vs. Ang-II+RMRP siRNA. Con, control; RMRP, RNA component of mitochondrial RNA processing endoribonuclease; si, small interfering; NC, negative control; miR, microRNA; $\alpha$-SMA, $\alpha$-smooth muscle actin; Ang II, angiotensin II.

and Ang-II-treated cardiac fibroblasts, suggesting that RMRP may serve an important role in cardiac fibroblast activation.
Previous studies investigating RMRP have focused on its role in carcinogenesis, including in lung cancer, gastric tumor 
and glioma (8-10). The upregulation of RMRP was indicated in left ventricle biopsies of patients with non-end-stage heart failure (12). Therefore, in the current study, the role of RMRP in cardiac fibroblasts activation was investigated further. The results demonstrated that RMRP knockdown by siRNA can inhibit Ang-II-induced proliferation, differentiation and collagen accumulation in cardiac fibroblasts, suggesting the promoting effect of RMRP on cardiac fibroblast activation.

lncRNAs can be used as competitive endogenous RNAs (ceRNAs) to regulate a variety of biological functions in cells. IncRNAs contain complements of the sequence domain of miRNAs, which allow them to act as natural sponges to bind with numerous miRNAs (29). IncRNA n379519 has been revealed to promote cardiac fibrosis in the post-infarct myocardium, by targeting miR-30 (28). PFL has also been indicated to contribute to cardiac fibrosis by acting as a ceRNA of let-7d (27). H19 was demonstrated to act as a ceRNA to mediate CTGF expression, by sponging miR-455, in cardiac fibrosis (30). In the present study, six miRNAs were predicted as targets of RMRP, according to the RNA sequence produced by the miRcode algorithm. Among them, miR-613 and miR-1 were reported to be closely associated with myocardial I/R injury and cardiac hypertrophy, respectively. miR-613 can suppress I/R-induced cardiomyocyte apoptosis by directly targeting PDCD10 and activating the PI3K/Akt pathway (20). Karakikes et al (21) demonstrated that normalization of miR-1 gene expression, which was downregulated in hypertrophy, reversed cardiac hypertrophy and attenuated pathological remodeling by simultaneously affecting a variety of processes that are associated with pathological hypertrophy and heart failure. In the present study, Ang-II downregulated miR-613 and miR-1 expression, while RMRP knockdown promoted miR-613 expression more so than miR-1. The results also demonstrated that RMRP shared complementary binding sites with miR-613 and this was validated using luciferase reporter and MS2 RIP assays. These results indicated that RMRP negatively regulated miR-613 expression in cardiac fibroblasts and allowed investigation into the role of miR-613 in the promoting effect of RMRP on cardiac fibroblast activation. The results revealed that the inhibition of miR-613 reversed the effect of RMRP knockdown on cardiac fibroblast activation. Collectively, RMRP promoted cardiac fibroblast activation by acting as a ceRNA for miR-613. Recently, Kong et al (31) demonstrated that the overexpression of RMRP aggravated hypoxia-induced injury in $\mathrm{H} 9 \mathrm{c} 2$ cardiomyocytes by sponging miR-206 to target ATG3 expression. RMRP may be involved in the pathological process of heart diseases by regulating cardiac fibroblast and cardiomyocyte function.

In conclusion, the results of the current study revealed that RMRP expression was upregulated in the fibrotic myocardium and activated cardiac fibroblasts during cardiac fibrosis, and RMRP promoted the activation of cardiac fibroblasts by competitively binding miR-613. These findings provided an advanced insight into the pathogenesis of lncRNAs in cardiac fibrosis, suggesting that RMRP inhibition may be a novel therapy for cardiac fibrosis. The prognostic significance of RMRP and miR-613 expression will aid in the prediction of heart-related disease phenotypes. Individual therapy targeting RMRP and miR-613, in combination, may provide more curative effects.

\section{Acknowledgements}

Not applicable.

\section{Funding}

No funding was received.

\section{Availability of data and materials}

All data generated and/or analyzed during this study are included in this published article.

\section{Authors' contributions}

SYZ and SHH designed the study. SYZ, SHH, SXG and YBW performed the experiments. PJ and FJL performed data analysis. SYZ and SHH wrote the main manuscript. SYZ, SHH and SXG contributed to manuscript revisions. All authors reviewed the manuscript.

\section{Ethics approval and consent to participate}

All animal experiments performed in the current study were approved by The Animal Care and Use Committee of Lanzhou University (Lanzhou, China).

\section{Patient consent for publication}

Not applicable.

\section{Competing interests}

The authors declare that they have no competing interests.

\section{References}

1. Gonzalez A, Schelbert EB, Diéz J and Butler J: Myocardial interstitial fibrosis in heart failure: Biological and translational perspectives. J Am Coll Cardiol 71: 1696-1706, 2018.

2. Weber KT, Sun Y, Tyagi SC and Cleutjens JP: Collagen network of the myocardium: Function, structural remodeling and regulatory mechanisms. J Mol Cell Cardiol 26: 279-292, 1994.

3. Hara H, Takeda $\mathrm{N}$ and Komuro I: Pathophysiology and therapeutic potential of cardiac fibrosis. Inflamm Regen 37: 13, 2017.

4. Djebali S, Davis CA, Merkel A, Dobin A, Lassmann T, Mortazavi A, Tanzer A, Lagarde J, Lin W, Schlesinger F, et al: Landscape of transcription in human cells. Nature 489: 101-108, 2012.

5. Creemers EE and van Rooij E: Function and therapeutic potential of noncoding RNAs in cardiac fibrosis. Circ Res 118: 108-118, 2016.

6. Hou J, Zhou C, Long H, Zheng S, Guo T, Wu Q, Wu H, Zhong T and Wang T: Long noncoding RNAs: Novel molecules in cardiovascular biology, disease and regeneration. Exp Mol Pathol 100: 493-501, 2016.

7. Rosenbluh J, Nijhawan D, Chen Z, Wong KK, Masutomi K and Hahn WC: RMRP is a non-coding RNA essential for early murine development. PLoS One 6: e26270, 2011.

8. Meng Q, Ren M, Li Y and Song X: LncRNA-RMRP acts as an oncogene in lung cancer. PLoS One 11: e0164845, 2016.

9. Shao Y, Ye M, Li Q, Sun W, Ye G, Zhang X, Yang Y, Xiao B and Guo J: LncRNA-RMRP promotes carcinogenesis by acting as a miR-206 sponge and is used as a novel biomarker for gastric cancer. Oncotarget 7: 37812-37824, 2016. 
10. Feng W, Li L, Xu X, Jiao Y and Du W: Up-regulation of the long non-coding RNA RMRP contributes to glioma progression and promotes glioma cell proliferation and invasion. Arch Med Sci 13: 1315-1321, 2017

11. Wang X, Peng L, Gong X, Zhang X, Sun R and Du J: LncRNA-RMRP promotes nucleus pulposus cell proliferation through regulating miR-206 expression. J Cell Mol Med 22: 5468-5476, 2018

12. Greco S, Zaccagnini G, Perfetti A, Fuschi P, Valaperta R, Voellenkle C, Castelvecchio S, Gaetano C, Finato N, Beltrami AP, et al: Long noncoding RNA dysregulation in ischemic heart failure. J Transl Med 14: 183, 2016.

13. Steinbusch MMF, Caron MMJ, Surtel DAM, Friedrich F, Lausch E, Pruijn GJM, Verhesen W, Schroen BLM, van Rhijn LW, Zabel B and Welting TJM: Expression of RMRP RNA is regulated in chondrocyte hypertrophy and determines chondrogenic differentiation. Sci Rep 7: 6440, 2017.

14. Cai SA, Hou N, Zhao GJ, Liu XW, He YY, Liu HL, Hua YQ, Li LR, Huang Y, Ou CW, et al: Nrf2 is a key regulator on puerarin preventing cardiac fibrosis and upregulating metabolic enzymes UGT1A1 in rats. Front Pharmacol 9: 540, 2018.

15. Li WQ, Li XH, Wu YH, Du J, Wang AP, Li D and Li YJ: Role of eukaryotic translation initiation factors 3a in hypoxia-induced right ventricular remodeling of rats. Life Sci 144: 61-68, 2016.

16. Livak KJ and Schmittgen TD: Analysis of relative gene expression data using real-time quantitative PCR and the 2(-Delta Delta $\mathrm{C}(\mathrm{T}))$ method. Methods 25: 402-408, 2001.

17. Chu W, Li C, Qu X, Zhao D, Wang X, Yu X, Cai F, Liang H, Zhang Y, Zhao X, et al: Arsenic-induced interstitial myocardial fibrosis reveals a new insight into drug-induced long QT syndrome. Cardiovasc Res 96: 90-98, 2012.

18. Chacar S, Farés N, Bois P and Faivre JF: Basic signaling in cardiac fibroblasts. J Cell Physiol 232: 725-730, 2017.

19. Porter KE and Turner NA: Cardiac fibroblasts: At the heart of myocardial remodeling. Pharmacol Ther 123: 255-278, 2009.

20. Wu Z, Qi Y, Guo Z, Li P and Zhou D: miR-613 suppresses ischemia-reperfusion-induced cardiomyocyte apoptosis by targeting the programmed cell death 10 gene. Biosci Trends 10: 251-257, 2016.

21. Karakikes I, Chaanine AH, Kang S, Mukete BN, Jeong D, Zhang S, Hajjar RJ and Lebeche D: Therapeutic cardiac-targeted delivery of miR-1 reverses pressure overload-induced cardiac hypertrophy and attenuates pathological remodeling. J Am Heart Assoc 2: e000078, 2013.
22. Jathar S, Kumar V, Srivastava J and Tripathi V: Technological developments in lncRNA biology. Adv Exp Med Biol 1008: 283-323, 2017.

23. Deniz E and Erman B: Long noncoding RNA (lincRNA), a new paradigm in gene expression control. Funct Integr Genomics 17: 135-143, 2017.

24. Nebbioso A, Tambaro FP, Dell'Aversana C and Altucci L: Cancer epigenetics: Moving forward. PLoS Genet 14: e1007362, 2018.

25. Tao H, Zhang JG, Qin RH, Dai C, Shi P, Yang JJ, Deng ZY and Shi KH: LncRNA GAS5 controls cardiac fibroblast activation and fibrosis by targeting miR-21 via PTEN/MMP-2 signaling pathway. Toxicology 386: 11-18, 2017.

26. Qu X, Du Y, Shu Y, Gao M, Sun F, Luo S, Yang T, Zhan L, Yuan Y, Chu W, et al: MIAT is a Pro-fibrotic long Non-coding RNA governing cardiac fibrosis in Post-infarct myocardium. Sci Rep 7: 42657, 2017.

27. Liang H, Pan Z, Zhao X, Liu L, Sun J, Su X, Xu C, Zhou Y, Zhao D, Xu B, et al: LncRNA PFL contributes to cardiac fibrosis by acting as a competing endogenous RNA of let-7d. Theranostics 8: 1180-1194, 2018.

28. Wang X, Yong C, Yu K, Yu R, Zhang R, Yu L, Li S and Cai S: Long noncoding RNA (lncRNA) n379519 promotes cardiac fibrosis in Post-infarct myocardium by targeting miR-30. Med Sci Monit 24: 3958-3965, 2018.

29. Yoon JH, Abdelmohsen K and Gorospe M: Functional interactions among microRNAs and long noncoding RNAs. Semin Cell Dev Biol 34: 9-14, 2014.

30. Huang ZW, Tian LH, Yang B and Guo RM: Long noncoding RNA H19 acts as a competing endogenous RNA to mediate CTGF expression by sponging miR-455 in cardiac fibrosis. DNA Cell Biol 36: 759-766, 2017.

31. Kong F, Jin J, Lv X, Han Y, Liang X, Gao Y and Duan X: Long noncoding RNA RMRP upregulation aggravates myocardial ischemia-reperfusion injury by sponging miR-206 to target ATG3 expression. Biomed Pharmacother 109: 716-725, 2019.

This work is licensed under a Creative Commons Attribution-NonCommercial-NoDerivatives 4.0 International (CC BY-NC-ND 4.0) License. 